\title{
Probing QCD with the ATLAS Detector
}

\author{
Yuri Kulchitsky ${ }^{1,2, *}$, on behalf of the ATLAS Collaboration \\ ${ }^{1}$ B.I.Stepanov Institute of Physics, National Academy of Sciences of Belarus, Minsk, Belarus \\ ${ }^{2}$ Joint Institute for Nuclear Research, Dubna, Russia
}

\begin{abstract}
Perturbative QCD calculations at next-to-next-to-leading-order are available for many processes since several years and can be rigorously tested with a large variety of final states. The latest results from the ATLAS Collaboration at the LHC (CERN) involving jets, dijets, photons in association with heavy flavors and vector bosons in association with jets, measured at center of mass energies of $\sqrt{s}=8$ and $13 \mathrm{TeV}$ are presented. All measured crosssections are compared to state-of-the art theory Monte Carlo predictions. The paper concludes with the results of jet-substructure studies at $13 \mathrm{TeV}$, in particular the measurement of the jet soft-drop mass.
\end{abstract}

\section{Introduction}

Precise measurements of jet cross-sections are crucial in understanding physics at hadron colliders. In quantum chromodynamics (QCD), jets are interpreted as resulting from the fragmentation of quarks and gluons produced in a short-distance scattering process. Jet cross-sections provide valuable information about the strong coupling constant, $\alpha_{s}$, and the structure of the proton. Inclusive jet and dijet events represent a background to many other processes at hadron colliders. The predictive power of fixed-order QCD calculations is therefore relevant in many searches for new physics. The ATLAS collaboration ${ }^{1}$ [1] at the LHC (CERN) [2] have measured inclusive jet cross-sections in $p p$ collisions at $\sqrt{s}=13 \mathrm{TeV}$ [3] and $8 \mathrm{TeV}$ [4]. Dijet production at the LHC has been measured at $\sqrt{s}=13 \mathrm{TeV}$ [3]. The production of isolated prompt photons in association with a heavy flavour (HF) jet containing a $b$ or $c$ hadron at $\sqrt{s}=8 \mathrm{TeV}$ [5] provides a testing ground for perturbative quantum chromodynamics (pQCD), the content of the proton and the treatment of heavy quarks (HQ) in matrix element (ME) and parton shower (PS) computations. Prompt photons, which refer to those not arising from hadron decays, are targeted by requiring that their signals are isolated. A measurement of the $W$ boson production cross section and the $W^{+} / W^{-}$cross-section ratio, both in association with jets, in $p p$ collisions at $\sqrt{s}=8 \mathrm{TeV}$ are presented in Ref. [6]. A measurement of the first jet substructure quantity was calculated at next-to-next-to-leadinglogarithm (NNLL) accuracy are presented in Ref. [7].

\section{Inclusive jet and dijet cross-sections at $\sqrt{s}=13 \mathrm{TeV}$}

This paper presents measurements of the inclusive jet and dijet cross-sections in $p p$ collisions at $\sqrt{s}=13 \mathrm{TeV}$ corresponding to an integrated luminosity of $3.2 \mathrm{fb}^{-1}$ [3]. The inclusive jet cross-sections are measured double-differentially as a function of the jet transverse momentum, $p_{\mathrm{T}}$, and absolute jet rapidity, $|y|$. The double-differential dijet production

\footnotetext{
*e-mail: Iouri.Kultchitski@cern.ch

${ }^{1}$ Copyright [2018] CERN for the benefit of the [ATLAS Collaboration]. Reproduction of this article or parts of it is allowed as specified in the CC-BY-4.0 license.
} 

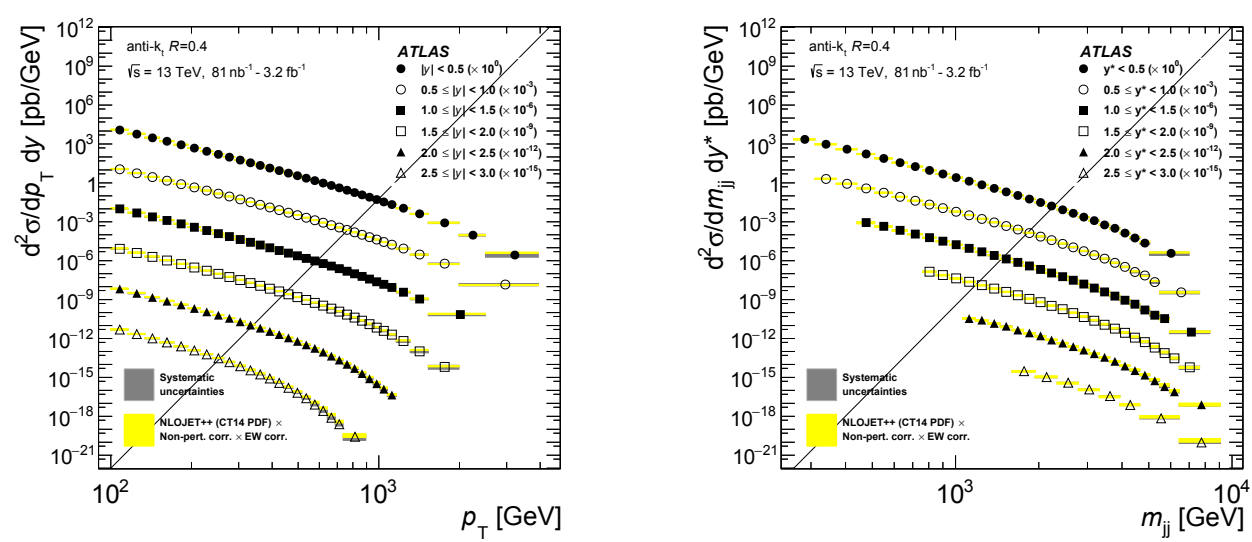

Figure 1. Inclusive (left) jet and (right) dijet cross-sections as a function of $p_{\mathrm{T}}$ for jet and $m_{\mathrm{jj}}$ for dijet and $|y|$, for anti- $k_{\mathrm{t}}$ jets with $R=0.4$ in $p p$ collisions at $\sqrt{s}=13 \mathrm{TeV}$. The statistical uncertainties are smaller than the size of the symbols used to plot the cross-section values. The dark gray shaded areas indicate the experimental systematic uncertainties. The data are compared to NLO pQCD predictions calculated using NLOJET++ with $p_{\mathrm{T}}^{\max }$ for jet and $p_{\mathrm{T}}^{\max } \exp \left(0.3 y^{*}\right)$ for dijet as the QCD scale and the CT14 NLO PDF set. The yellow shaded areas indicate the predictions with their uncertainties. At low and intermediate $p_{\mathrm{T}}$ bins the experimental systematic uncertainties are comparable to the theory uncertainties (drawn on top) and therefore are barely visible. In most $m_{\mathrm{jj}}$ bins the experimental systematic uncertainty is smaller than the theory uncertainties and is therefore not visible. Taken from Ref. [3].

cross-sections are presented as a function of the invariant mass of the dijet system, $m_{\mathrm{jj}}$, and as a function of half the absolute rapidity separation between the two highest- $p_{\mathrm{T}}$ jets satisfying $|y|<3$, denoted $y^{*}$. Jets are reconstructed using the anti- $k_{\mathrm{t}}$ jet clustering algorithm with a radius parameter value of $R=0.4$. The measurements cover the kinematic region of $0.1<p_{\mathrm{T}}<3.5 \mathrm{TeV}$ and $|y|<3$ for the inclusive jet cross-section, and of $0.3<m_{\mathrm{jj}}<9 \mathrm{TeV}$ and $y^{*}<3$ for the dijet cross-section. NLO pQCD predictions calculated using several parton distribution function (PDF) sets, corrected for electroweak (EW) and non-perturbative (NP) effects, are quantitatively compared to the measurement results. In addition, the inclusive jet cross-sections are compared to the recently published complete next-to-next-to-leading-order (NNLO) pQCD calculation. The measured double-differential inclusive jet cross-sections are shown in Fig. 1(left) as a function of $p_{\mathrm{T}}$ for the 6 jet rapidity bins, and the measured doubledifferential dijet cross-sections are shown in Fig. 1(right) as a function of $m_{\mathrm{jj}}$ for the $6 y^{*}$ bins. The NLO pQCD predictions using the CT14 PDF set corrected for NP and EW effects are also shown in Fig. 1. The ratios of the NLO pQCD predictions to the measured inclusive jet cross-sections as a function of $p_{\mathrm{T}}$ in the 6 jet $|y|$ bins are shown in Fig. 2 for the CT14, MMHT2014, NNPDF3.0, HERAPDF2.0, ABMP16 PDF sets. No significant deviation of the data points from the predictions is seen; the NLO pQCD predictions and data agree within uncertainties. In the forward region $(|y|>2)$ there is a tendency for the NLO pQCD prediction using the CT14, MMHT2014 and NNPDF3.0 PDF sets to overestimate the measured cross-section in the high $p_{\mathrm{T}}$ range, although the difference from data does not exceed the range covered by the experimental and theoretical uncertainties. A quantitative comparison of the measurements to fixed-order NLO QCD calculations shows overall fair agreement when considering jet cross-sections in individual jet $|y|$ bins independently. In the inclusive jet measurement, a significant tension between data and theory is observed when considering data points from all jet $p_{\mathrm{T}}$ and $|y|$ regions. No significant differences between the inclusive jet cross-sections and the fixed-order NNLO QCD calculations corrected for NP and EW effects 

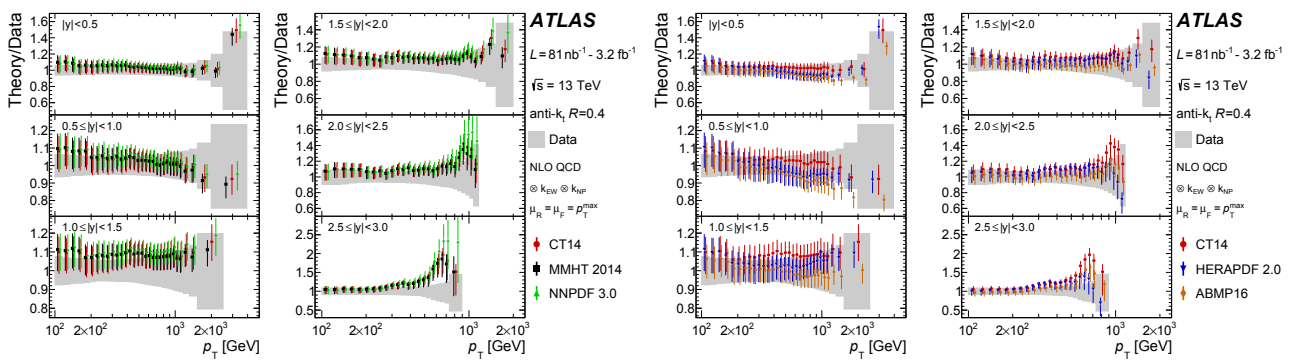

Figure 2. Comparison of the measured inclusive jet cross-sections and the NLO pQCD predictions shown as the ratios of predictions to the measured cross-sections in $p p$ collisions at $\sqrt{s}=13 \mathrm{TeV}$. The ratios are shown as a function of the jet $p_{\mathrm{T}}$ in $6|y|$ bins for anti- $k_{\mathrm{t}}$ jets with $R=0.4$. The predictions are calculated using NLOJET++ with different PDF sets (left) [CT14, MMHT2014, NNPDF3.0] and (right) [CT14, HEPAPDF3.0, ABMP16]. The NP and EW corrections are applied. The gray bands show the total data uncertainty including both the systematic and statistical uncertainties. Taken from Ref. [3].
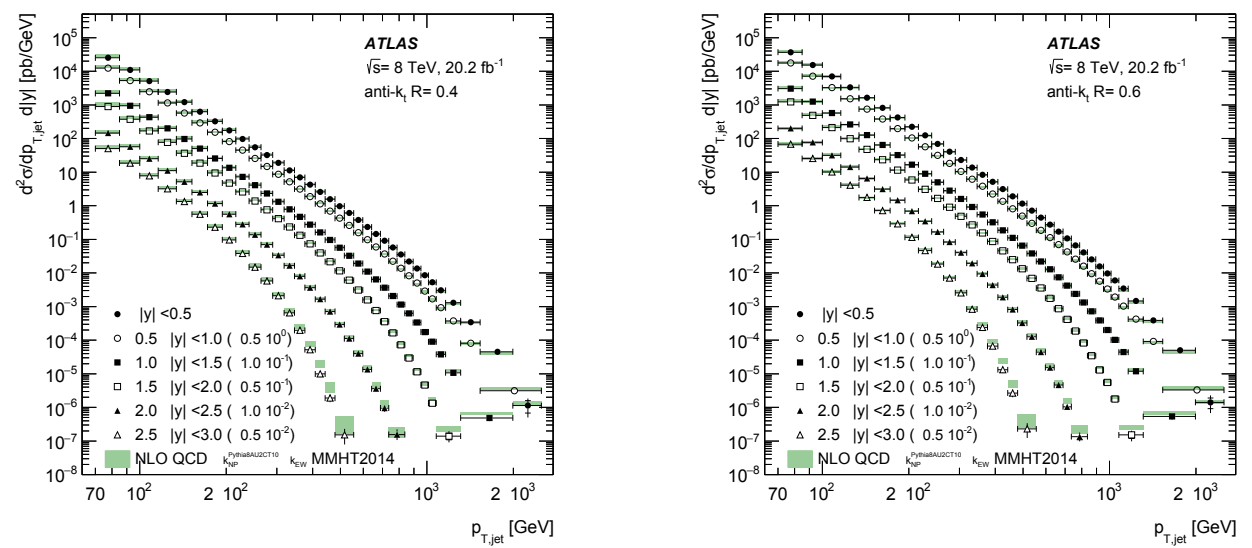

Figure 3. Inclusive jet cross-section as a function of jet $p_{\mathrm{T}}$ in bins of jet $|y|$ in $p p$ collisions at $\sqrt{s}=8$ $\mathrm{TeV}$. The results are shown for jets identified using the anti- $k_{\mathrm{t}}$ algorithm with (left) $R=0.4$ and (right) $R=0.6$. For better visibility the cross-sections are multiplied by the factors indicated in the legend. The data are compared to the NLO QCD prediction with the MMHT2014 PDF set. The error bars indicate the statistical and the systematic uncertainties in the measurement added in quadrature. The statistical uncertainty is shown separately by the inner vertical line. Taken from Ref. [4].

are observed when using $p_{\mathrm{T}}^{\mathrm{jet}}$ as the QCD scale. The NLO pQCD predictions using $p_{\mathrm{T}}^{\max }$ as the scale overestimate the measured inclusive jet cross-sections.

\section{Inclusive jet cross-sections at $\sqrt{s}=8 \mathrm{TeV}$}

The measured double-differential inclusive jet cross-sections at $\sqrt{s}=8 \mathrm{TeV}$ are shown in Fig. 3 as a function of the jet $p_{\mathrm{T}}$ for anti- $k_{\mathrm{t}}$ jets with $R=0.4$ and $R=0.6$ for each jet $|y|$ bin [4]. The cross-section covers 11 orders of magnitude in the central $|y|$ region and 9 orders of magnitude in the forward $|y|$ region. Jet transverse momenta above $p_{\mathrm{T}}=2 \mathrm{TeV}$ are observed. In the most forward region the jet $p_{\mathrm{T}}$ reaches about $0.5 \mathrm{TeV}$. The measurement is compared to an NLO QCD prediction using the MMHT2014 PDF set based on NLOJET++ corrected for NP and EW effects. This theory prediction describes the gross features in the data. The ratio of NLO QCD calculations to data corrected for NP and EW effects for various PDF sets is shown in Fig. 4(left) for anti- $k_{\mathrm{t}}$ jets with $R=0.4$. [4]. At low $p_{\mathrm{T}}$ the level of agreement 

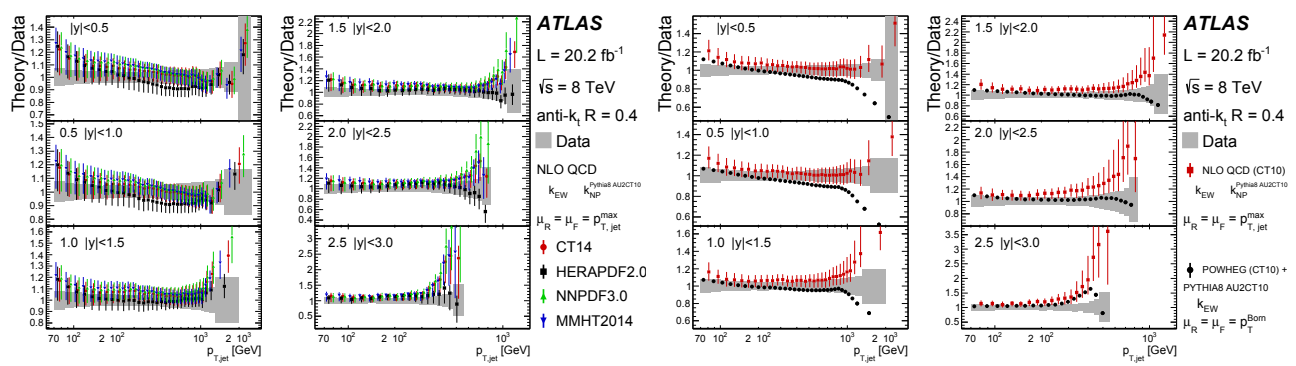

Figure 4. (Left) Ratio of the inclusive jet cross-section predicted by NLO QCD corrected for NP and EW effects to the cross-section in data as a function of the jet $p_{\mathrm{T}}$ in each jet $|y|$ bin in $p p$ collisions at $\sqrt{s}=8 \mathrm{TeV}$. Shown are the predictions for various PDF sets for anti- $k_{\mathrm{t}}$ jets with $R=0.4$. The points are offset in jet $p_{\mathrm{T}}$ for better visibility. The error bars indicate the total theory uncertainty. The gray band shows the total uncertainty in the measurement. (Right) Ratio of the inclusive jet cross-section predicted by the Powheg MC event generator with respect to the cross-section in data as a function of the jet $p_{\mathrm{T}}$ in each jet $|y|$ bin for anti- $k_{\mathrm{t}}$ jets with $R=0.4$. Only the nominal values of this ratio are indicated. Also shown is the prediction by NLO QCD corrected for NP effects, where the error bars indicate the total theory uncertainty. EW corrections are applied for both theory predictions and the CT10 PDF set is used. Taken from Ref. [4].

is very sensitive to NP effects. When using Pythia8 as the nominal NP correction, the NLO QCD prediction is typically about $10-20 \%$ above the data at low $p_{\mathrm{T}}$, whereas the NLO QCD prediction corrected with HeRwIG++ follows the data well for jets with $R=0.4$, while it is $5-10 \%$ below the data for jets with $R=0.6$. The comparisons of the PowHEg predictions with the measurement for jets with $R=0.4$ are shown in Fig. 4(right) as a function of the jet $p_{\mathrm{T}}$ in jet $|y|$ bins. The measurements are also compared to the NLO QCD prediction using the CT10 PDF set and corrected for NP effects with the same Monte Carlo (MC) generator configuration as was used for PowHEg. Strong tension between data and theory is observed when considering data points from all jet $p_{\mathrm{T}}$ and $|y|$ regions, with a full treatment of the correlations. This tension can be reduced, but not completely resolved, using alternative correlation scenarios for the experimental and theoretical systematic uncertainties [4].

\section{Differential cross-sections of $\gamma$ with b or $\mathbf{c}$ jet at $\sqrt{s}=8 \mathrm{TeV}$}

Prompt photons $(\gamma)$ can be used as a colourless non-hadronizing probe of parton dynamics that yields a clean experimental signature. Processes containing final state $b$ or $c$ quarks play an important role in many LHC physics analyses and therefore the accuracy of the description of this HF content of the proton must be investigated. HF jets are defined as jets which contain either a $b$ or $c$ hadron. In Fig. 5 the values for the measured differential $\gamma+c$ cross sections are plotted and in Fig. 6 the ratios of $\gamma+b$ and $\gamma+c$ for central and forward regions are given, with the relevant theory predictions [5]. In general, considering the LO predictions in pQCD, those from SHERPA agree well with the measured values and provide a better description of the data than those from Pythia. Comparisons of the $\gamma+c$ measurement to NLO+PS predictions from MadGraPh5_AMC@NLO in the 5F scheme using NNPDF3.1NLO with $0.26 \%$ of the intrinsic charm (IC) and CT14NNLO are shown in Fig. 5. The predictions are found to agree with the data within the uncertainties across the entire $E_{\mathrm{T}}^{\gamma}$ range. However, those using the BHPS or the fitted charm PDF sets predict higher cross-section values in the forward region at high $E_{\mathrm{T}}^{\gamma}$, above $105 \mathrm{GeV}$, than those using the nominal PDF sets. As shown in Fig. 6(left), the $4 F$ and $5 F$ NLO predictions for the cross-section ratios consistently overestimate the data for $E_{\mathrm{T}}^{\gamma}>65 \mathrm{GeV}$; the $5 F$ predictions are at the edge of agreement with the measured values within uncertainties. SHERPA, which generates additional partons in the ME and uses a 

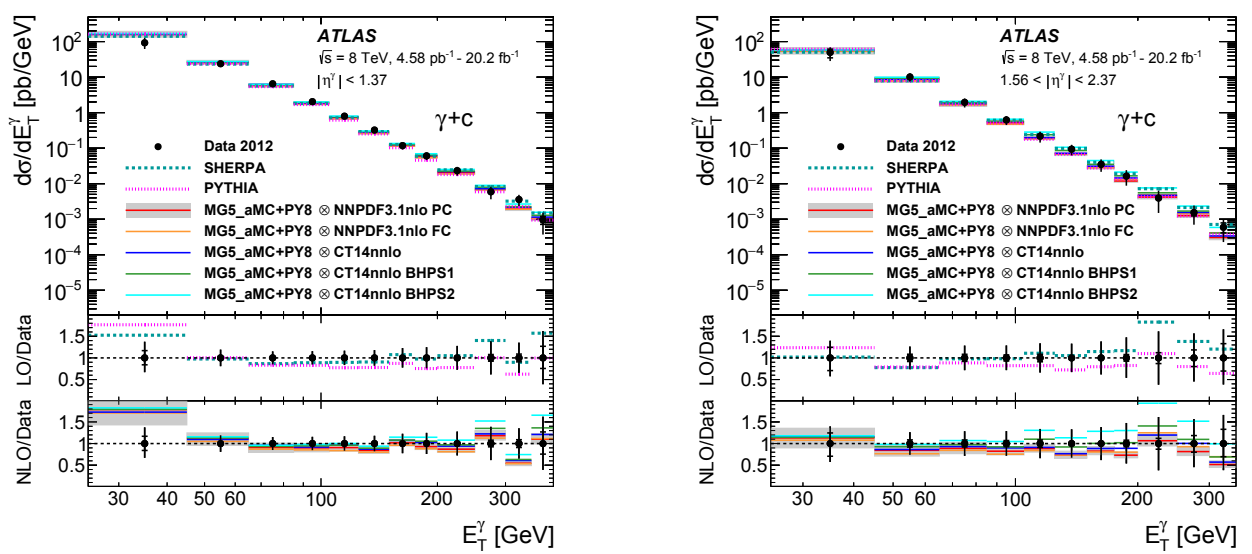

Figure 5. Differential cross sections as a function of the photon transverse energy, $E_{\mathrm{T}}^{\gamma}$, for $\gamma+c$ in (left) the central and (right) the forward regions in $p p$ interactions at $\sqrt{s}=8 \mathrm{TeV}$. The statistical uncertainty is represented as horizontal marks on the error bars of the data points, while the total measurement uncertainty is represented by the complete length of the error bars. The MG5_AMC+PY8 label in the legend refers to the MaDGraph5_AMC@NLO calculations interfaced to Pythia8. The $5 F$ and $4 F$ labels in the legend refer to PDF sets with five and four quark flavours, respectively. The PC and FC labels in the legend refer to perturbative charm and fitted charm PDF sets, respectively. All of the predictions for $\gamma+c$ use 5F PDF sets. The theoretical uncertainty in the MADGRAPH5_AMC@NLO predictions is displayed for a single PDF set since it is similar for each of the PDF sets. The SHERPA and Pythia cross sections are not normalized to data and no uncertainties are provided for them. Taken from Ref. [5].

massive $5 F$ scheme, provides a better description of the measured cross sections and crosssection ratios than MADGRAPH5_AMC@NLO in either the 5F or 4F scheme. The predicted values for the cross-section ratios, shown in Fig. 6(right), are smaller for the predictions using the BHPS or the fitted charm sets than for those using the nominal sets. This is the expected behaviour of the IC contributions from these PDF sets in the theory predictions. The predictions with the BHPS2 PDF set (2.1\% of the IC) deviate the most from those using the nominal PDF sets, by about a factor 1.5 , while those using the BHPS1 $(0.6 \%$ of the IC) and the fitted charm PDF sets give intermediate values. The precision of the data is comparable to the size of these deviations in the predictions. For the $\gamma+b$ final state, the best description of the data is provided by the SHERPA predictions, which include up to three additional partons and are computed in the massive $5 F$ scheme. The NLO predictions underestimate the data in the highest $E_{\mathrm{T}}^{\gamma}$ intervals measured. The $5 F$ scheme of the theoretical calculations provides a better description of the data than the $4 F$ scheme. For the $\gamma+c$ final state, which exhibits larger measurement uncertainties, all the predictions are in agreement with the data. Differences of about the size of the measurement uncertainties are seen between the predictions using PDF sets with IC contributions and those without. These measured cross sections provide a test of pQCD calculations with HQs and are sensitive to the $b$ and $c$ quark PDFs.

\section{Differential cross-sections of $\mathbf{W}+$ jets and $\mathbf{W}^{+} / \mathbf{W}^{-}$ratios at $\sqrt{s}=8 \mathrm{TeV}$}

Using data corresponding to an integrated luminosity of $20.2 \mathrm{fb}^{-1}$ at $\sqrt{s}=8 \mathrm{TeV}$, the results for $W+$ jets production in final states containing one electron and missing transverse momentum, focusing on events with one or two additional jets are presented [6]. The data are measured for $W$ production as well as for $W^{+}$and $W^{-}$production and the cross-section ratio of $W^{+} / W^{-}$as a function of the number of jets $\left(N_{\text {jets }}\right)$. For events with at least one jet, the differential cross sections are shown as a function of the scalar sum of the transverse 

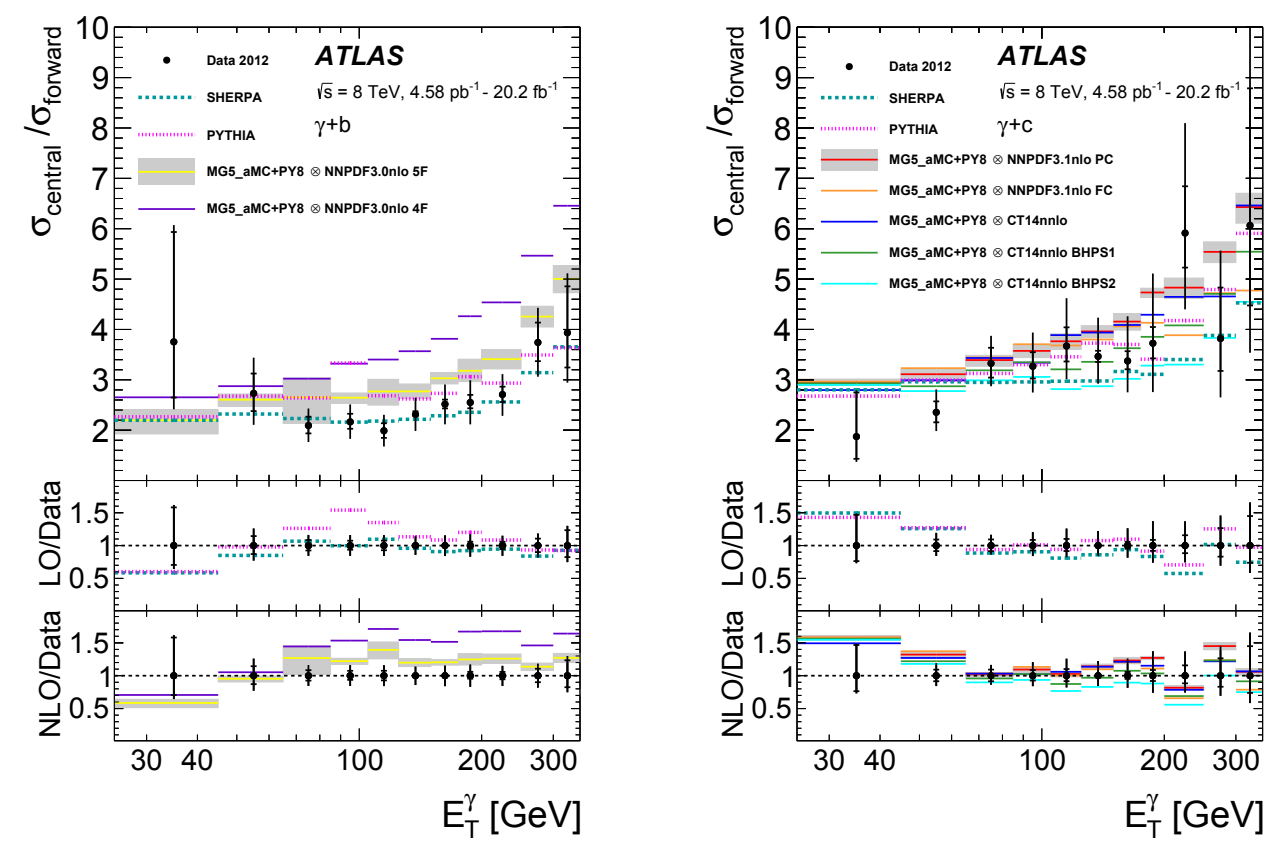

Figure 6. Cross-section ratios of the central region, $\left|\eta^{\gamma}\right|<1.37$, to the forward region, $1.56<\left|\eta^{\gamma}\right|<$ 2.37, as a function of the photon transverse energy, $E_{\mathrm{T}}^{\gamma}$, for (left) $\gamma+b$ and (right) $\gamma+c$. The statistical uncertainty is represented as horizontal marks on the error bars of the data points, while the total measurement uncertainty is represented by the complete length of the error bars. The MG5_AMC+PY8 label in the legend refers to the MadGraph5_AMC@NLO calculations interfaced to Pythia8. The 5F and $4 F$ labels in the legend refer to PDF sets with five and four quark flavours, respectively. The PC and FC labels in the legend refer to perturbative charm and fitted charm PDF sets, respectively. All of the predictions for $\gamma+c$ use 5F PDF sets. The theoretical uncertainty in the MADGraPH5_AMC@NLO predictions is displayed for a single PDF set since it is similar for each of the PDF sets, except for NNPDF3.1NLo FC for which the total uncertainty is similar to that in NNPDF3.1NLo PC at a value of $25 \mathrm{GeV}$ in $E_{\mathrm{T}}^{\gamma}$, but rises steadily relative to it to be a factor of three larger at $350 \mathrm{GeV}$. No uncertainties are provided for Sherpa and Pythia. Taken from Ref. [5].

momenta of electron, neutrino and jets $\left(H_{\mathrm{T}}\right)$, the $p_{\mathrm{T}}$ of the $W$ boson, and the $p_{\mathrm{T}}$ and rapidity of the most energetic jet (leading jet). These observables are sensitive to higher-order terms in the prediction as well as the PDFs. For events with at least two jets, the differential cross sections are shown for $W$ boson production only and include distributions as a function of the $p_{\mathrm{T}}$ and rapidity of the second leading jet, the dijet angular separation, and the dijet invariant mass. These observables are sensitive to hard parton radiation at large angles and different ME/PS matching schemes. The differential cross section for $W$ production and the ratio of $W^{+}$and $W^{-}$as a function of $H_{\mathrm{T}}$ are shown in Fig. 7 for $N_{\text {jets }} \geq 1$. The $H_{\mathrm{T}}$ distribution is a very important test of $\mathrm{pQCD}$ as the higher values are sensitive to higher jet multiplicities and topologies such as $q q \rightarrow q q^{\prime} W$. The LO predictions of Sherpa and Alpgen, which both include multiple jets in the ME calculation describe the data best, although these predictions have large theoretical uncertainties. The BLACKHAT+ShERPa predictions underestimate the data at large values of $H_{\mathrm{T}}$. This is expected since, at these large values of $H_{\mathrm{T}}$, contributions from additional jets are important, which are only partially present in this calculation. The predictions from the BLACKHaT+Sherpa exclusive sums method and from the NNLO $N_{\text {jetti }}$ calculation, which include an additional jet emission at NLO, provide better agreement with 

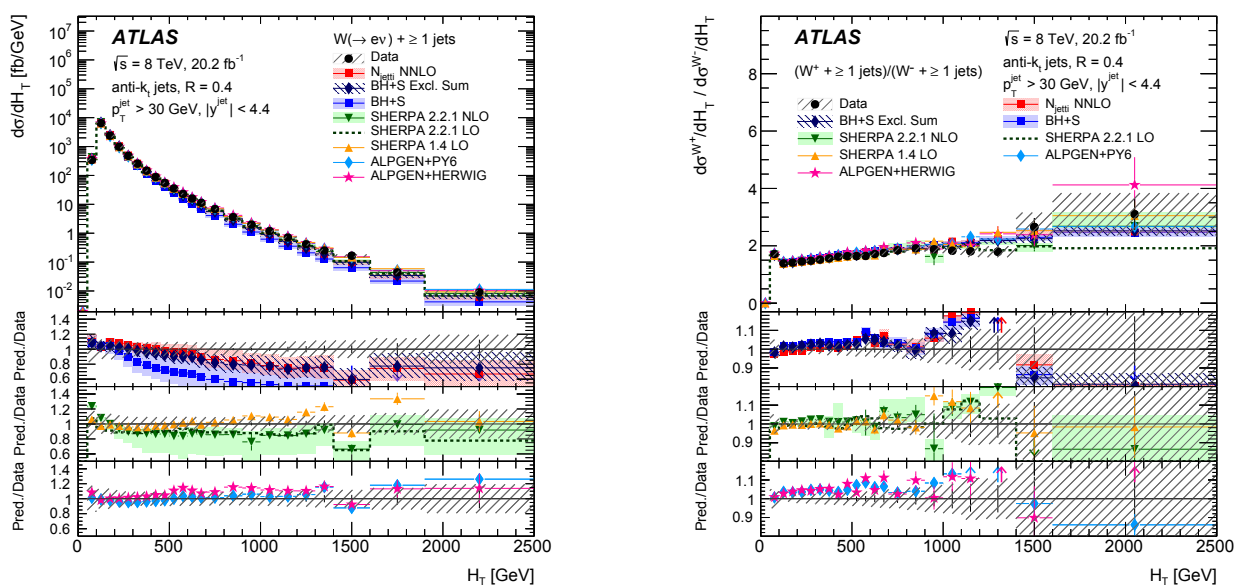

Figure 7. Differential cross sections for the production of (left) $W$ bosons and (right) the $W^{+} / W^{-}$ratio as a function of $H_{\mathrm{T}}$ for events with $N_{\text {jets }} \geq 1$. The last bin in the left figure includes values beyond the shown range. For the data, the statistical uncertainties are indicated as vertical bars, and the combined statistical and systematic uncertainties are shown by the hatched bands. The uppermost panel in plot shows the differential cross sections. The lower panels show the ratios of the predictions to the data. The arrows on the lower panels indicate points that are outside the displayed range. Taken from Ref. [6].

the data. At the largest measured values of $H_{\mathrm{T}}$, where the measured cross section is small, the total experimental uncertainty in the $W^{+} / W^{-}$cross-section ratio increases due to larger statistical uncertainties in the data and some systematic uncertainties that do not fully cancel out in the ratio. Overall, the measured distributions show that NNLO and NLO predictions are able to describe the data. However, at high transverse momenta, large jet rapidities, or large dijet angular separations, many of these predictions underestimate or overestimate the cross sections. In many places, multi-leg LO generators, such as AlPGEN and SHERPA, which consider a larger number of parton emissions from the ME calculation, model the data best, although with large theoretical uncertainties. The $H_{\mathrm{T}}$, jet rapidity, and dijet invariant mass distributions are in general the least well described, suggesting that better modelling of events with energetic jets as well as jets with large rapidities is needed.

\section{Measurement of the soft-drop jet mass at $\sqrt{s}=13 \mathrm{TeV}$}

Calculations of jet substructure observables that are accurate beyond LL accuracy have recently become available. In the Ref. [7] the normalized, differential cross-section is measured as a function of $\log _{10}\left(\rho^{2}\right)$, where $\rho$ is the ratio of the soft-drop mass to the ungroomed jet $p_{\mathrm{T}}$. This quantity is measured in dijet events from $32.9 \mathrm{fb}^{-1}$ of $\sqrt{s}=13 \mathrm{TeV} p p$ collisions. The soft-drop procedure acts on the clustering history of a sequential recombination jet algorithm. In these algorithms, all inputs to jet-finding start as a proto-jet and are combined pairwise using a distance metric in $y-\phi$ space. When the smallest distance is above some threshold $R$, the algorithm terminates and the remaining proto-jets are the final jets. The clustering history is the sequence of pairwise combinations that lead to a particular jet. The soft-drop algorithm starts by re-clustering an anti- $k_{\mathrm{t}}$ jet's constituents. Next, the clustering tree is traversed from the latest branch to the earliest and at each node the following criterion is applied to proto-jets $j_{1}$ and $j_{2}: \frac{\min \left(p_{\mathrm{T}_{\mathrm{j} 1}}, p_{\mathrm{T}_{2} \mathrm{j}_{2}}\right)}{p_{\mathrm{T}_{1} 1}+p_{\mathrm{T}_{2}}}>z_{\text {cut }}\left(\frac{\Delta R_{12}}{R}\right)^{\beta}$, where $p_{\mathrm{T}}$ is the momentum of a jet transverse to the beam pipe, $z_{\text {cut }}$ and $\beta$ are parameters, and $\Delta R_{12}=\sqrt{\Delta y^{2}+\Delta \phi^{2}}$ is the distance in $y-\phi$ between the proto-jets. The data are unfolded to correct for detector 

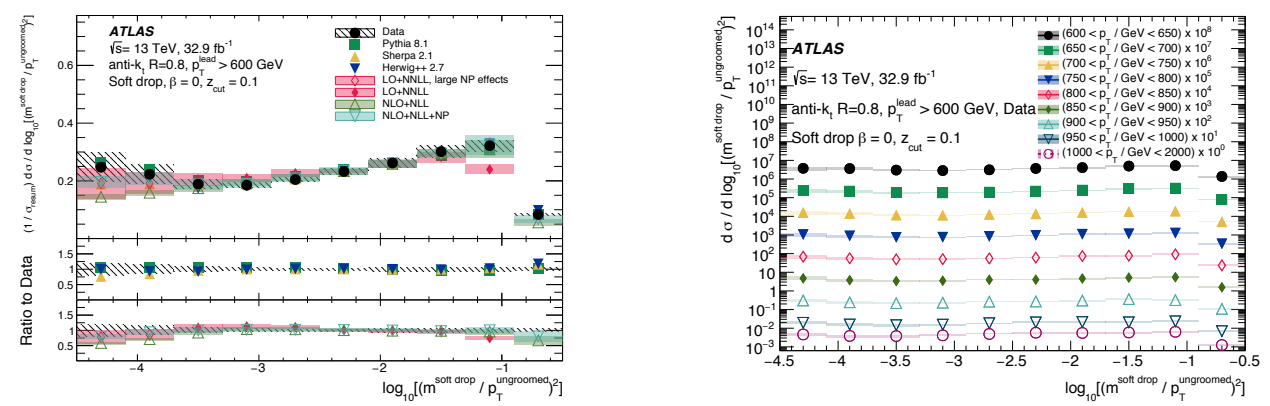

Figure 8. (Left) The unfolded $\log _{10}\left(\rho^{2}\right)$ distribution for anti- $k_{\mathrm{t}} R=0.8$ jets with $p_{\mathrm{T}}^{\text {lead }}>600 \mathrm{GeV}$, after the soft drop algorithm is applied for $\beta=0$, in data compared to Pythia, Sherpa, and Herwig ++ particle-level, and NLO+NLL+NP and LO+NNLL theory predictions. The LO+NNLL calculation does not have NP corrections; the region where these are expected to be large is shown in a open marker, while regions where they are expected to be small are shown with a filled marker. The distributions are normalized to the integrated cross section, $\sigma_{\text {resum }}$, measured in the resummation region, $-3.7<$ $\log _{10}\left(\rho^{2}\right)<-1.7$. The NLO+NLL+NP cross-section in this resummation regime is $0.14 \mathrm{nb}$ for $\beta=0$. (Right) The unfolded $\log _{10}\left(\rho^{2}\right)$ distribution in data for each of the $p_{\mathrm{T}}$ bins in the analysis, overlaid with a multiplicative factor indicated in the legend. Taken from Ref. [7].

effects and compared to precise QCD calculations and LL particle-level MC simulations and are shown in Fig. 8(left). They are compared to the predictions of the Pythia, Sherpa, and Herwig++ generators, as well as the NLO+NLL and the LO+NNLL. The NLO+NLL calculation includes NP corrections based on the average of various MC models with NP effects turned on and off; the envelope of predictions is added as an uncertainty. The LO+NNLL predictions do not contain NP effects, but the open makers in Fig. 8(left) indicate where NP are expected to be large. The $\log _{10}\left(\rho^{2}\right)$ distribution in data for $\beta=0$ and each $p_{\mathrm{T}}$ bin is shown in Fig. 8(right). There is no strong dependence of the shape on $p_{\mathrm{T}}$.

\section{Summary}

The latest results from the ATLAS collaboration at the LHC (CERN) involving jets, dijets, photons in association with HF jets and vector bosons in association with jets, measured at $\sqrt{s}=8$ and $13 \mathrm{TeV}$ are obtained. The first measurement of $\gamma+\mathrm{HF}$ jet at the LHC are presented. All measured cross-sections are compared to state-of-the art theory predictions.

\section{References}

[1] ATLAS Collaboration, JINST 3, S08003 (2008).

[2] L. Evans and P. Bryant (editors), JINST 3, S08001 (2008).

[3] ATLAS Collaboration, JHEP 1805, 195 (2018)

[4] ATLAS Collaboration, JHEP 1709, 020 (2017)

[5] ATLAS Collaboration, Phys. Lett. B 776, 295 (2018)

[6] ATLAS Collaboration, JHEP 1805, 077 (2018)

[7] ATLAS Collaboration, Phys. Rev. Lett. 121, 092001 (2018) 